\title{
A New Signalsome Containing Cytohesin-2 and CCDC120 Controls Neurite Outgrowth
}

\author{
Corresponding author: \\ Junji Yamauchi, Ph.D. \\ Department of Pharmacology \\ National Center for Child Health and Development \\ Setagaya, Tokyo157-8535, Japan. \\ Phone:81-3-5494-7120 (Ext. 4670) \\ Fax: 81-3-5494-7057 \\ yamauchi-j@ncchd.go.jp
}

Tomohiro Torii ${ }^{1}$, and Junji Yamauchi ${ }^{12^{*}}$

${ }^{1}$ Department of Pharmacology, National Center for Child Health and Development, Setagaya, Tokyo157-8535, Japan, ${ }^{2}$ Graduate School of Medical and Dental Sciences, Tokyo Medical and Dental University, Bunkyo, Tokyo 113-8510, Japan

E-mail: torii-t@ncchd.go.jp

E-mail: yamauchi-j@ncchd.go.jp

\begin{abstract}
Many functions in the nervous system depend on neural morphological changes including the cell polarity and neurite outgrowth and propagation of action potentials through networks of neuron-neuron and neuron-glia. Previous studies demonstrated that these associations are necessary for small GTPases activation in neurons. Especially, it is well known that small GTPases play a key role in neurite outgrowth, which is one of the most important events in neuronal development. Recent studies also have identified and characterized that molecular mechanisms of membrane trafficking and actin cytoskeleton formation/reorganization in migrating cells mediated by ADP-ribosylation factor 6 (Arf6) and its regulator cytohesin-2 are required for cell migration and neurite outgrowth. However, it have not completely established yet whether Arf6 activation is necessary or not in molecular mechanisms of neurite outgrowth. Our recent studies first identified and characterized that the previous unknown functional coiled-coil containing protein 120 (CCDC120) binds to cytohesin-2 and coordinately regulates neurite outgrowth in neuronal cells. This review summarizes the process that has been made in our understanding about the necessity of Arf6 activation in neurite outgrowth and the signaling transduction including cytohesin-2-CCDC120 complex in neuronal cells.
\end{abstract}

Keywords: Arf6; cytohesin-2; CCDC120; neurite outgrowth; axonal transport; 


\section{INTRODUCTION}

In the developing nervous system, neuronal cells continuously change their morphology and undergo neurite outgrowth, axon branching, axon navigation, and synaptogenesis to form neural networks (Craig and Banker 1994, da Silva and Dotti 2002, Gallo 2011). In particularly, it is well established that neurite outgrowth is mediated by a complicated process and involves various dynamic molecular mechanisms (Mattila and Lappalainen 2008). Increasing studies demonstrated that a number small GTPases, including members of the Ras, Rho (Govek et al. 2005), Arf (Sabe 2003, D'Souza-Schorey and Chavrier 2006), Arf-like (Arl) (Burd et al. 2004, Kahn et al. 2006), and Rab (Stenmark 2009, Yap and Winckler 2012, Villarroel-Campos et al. 2014) GTPase families regulate actin cytoskeleton formation/reorganization, membrane trafficking, or neuronal vesicle's axonal transport. These intracellular morphological changes contribute to neurite outgrowth, dendritic spine formation, or neuronal migration as subsequent neural functions, respectively.

Membrane proteins accumulate on surface of cell soma, growth cone, dendrite spine, and axonal surface in neurons. Theses signal molecules localizations are required for transport mediated by motor proteins such as kinesin superfamily protein (KIF) (Hirokawa 1997, Miki et al. 2001, Hancock et al. 2014), dynein (Hancock et al. 2014), Rab (Villarroel-Campos et al. 2014), and Arf families (Donaldson 2003, D'Souza-Schorey and Chavrier 2006, Kahn et al. 2006). Especially, many studies focus on Rab and Arf families, which control membrane/vesicle trafficking among intracellular regions (plasma membrane, endosomes, trans Golgi network (TGN), or endoplasmic reticulum (ER) in cells, respectively
(Stenmark 2009). In addition, it was cleared that the crosstalk between multiple GTPases (Arfs-Rabs (Chesneau et al. 2012, Kobayashi et al. 2014a, Miyamoto et al. 2014, Pelletán et al. 2015) or Rabs-Rabs (Yamamoto et al. 2010, Kobayashi et al. 2014b)) in a signaling pathway is responsible for membrane trafficking, vesicle neurite outgrowth, or cell differentiation. For example, Rab protein associates with activation of Arf protein and regulate actin remodeling endocytic regulation, ligand-specific endocytic regulation, and endosomal regulation of cell adhesion molecule processing and trafficking (Stenmark 2009). Recent study also demonstrates that recycling endosome or late endosome translocation also plays important role for neurite outgrowth in neurons. The repeated ER-endosome contact that is mediated by Rab7 accelerate neurite outgrowth (Raiborg et al. 2015). Collectively, these associations with small GTPases Rho family, Rab family, or Arf family regulate various neural functions in the nervous systems, respectively.

Although signal transduction pathway through Arf6-cytohesin-2 in migrating cells has been established as previous studies, it's neural function remains poorly understood. We have demonstrated that cytohesin-2 and its binding partner coordinately regulates acting through Arf6, neurite outgrowth (Yamauchi et al. 2009, Torii et al. 2012, Torii et al. 2014). In this review, we consider how neurite outgrowth is influenced by Arf6 activation and also whether the complex formation between cytohesin-2 and a new cytohesin-2 binding partner CCDC120 associate with neurite outgrowth in neuronal cells.

1. Roles of Arf6 GTPase in alteration of cellular morphology and cell migration Arf6 is a member of the 
A new signalsome containing cytohesin-2 and CCDC120 controls neurite outgrowth

ADP-ribosylation factor of GTP-binding proteins. Mammalian Arf family consists of three classes (class I (Arf1 and Arf2 and/or Arf3), class II (Arf4 and Arf5), and class III (Arf6)). Especially, Arf6 also regulate actin cytoskeleton dynamics, membrane trafficking, endocytosis and recycling of membrane proteins in the cells (Donaldson 2003, Sabe 2003, D'Souza-Schorey and Chavrier 2006, Kahn et al. 2006) and in the nervous system (Jaworski 2007). Arfs as well as Ras and Rho GTPases also acts as molecular switches that are active when bound to GTP and are inactive when bound to GDP. The biological switching is regulated by Arf6 specific guanine nucleotide exchange factors (GEFs) and GTPase-activating proteins (GAPs), strictly control the Arf6 guanine nucleotide-binding state and necessary for various physiological phenomena. Arf6 GEFs also define the strength and/or the cellular compartment to activate Arf6 by integrating the upstream signals.

Activated GTP-bound form Arf6 regulates phospholipase D (PLD), phosphatidylinositol 4-phosphate 5-kinase (PIP5K), and Rac1 [Figure 1B (Santy and Casanova 2001, Palacios and D'Souza-Schorey 2003)]. Especially, it has been understood that Arf6-mediated Rac activation regulates cell migration and forms lamellipodia at the leading edge in non-neuronal cells (Santy and Casanova 2001,
Figure 1

A

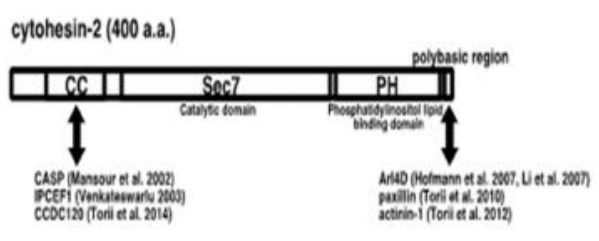

B

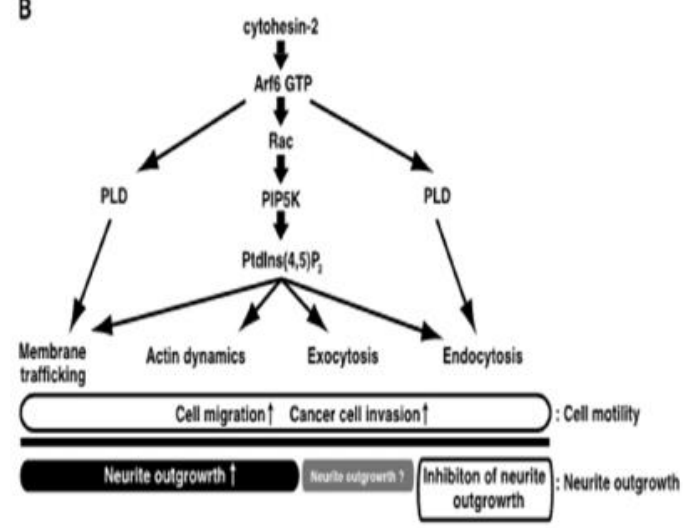

Figure 1.

A. Schematic illustration of mouse cytohesin-2. The schematic structures of full-length cytohesin-2 and its domains are illustrated $(\mathrm{CC}$; coiled coil domain, Sec7, PH; Pleckstrin homology). Previous studies demonstrated that CASP/Cybr/CYTIP, IPCEF1, and CCDC120 bind to CC region of cytohesin-2 and cytohesin-2 also interacts with Ar14D, paxillin, and actinin-1 through its C-terminal polybasic region.

B. A model for active Arf6-induced signal transduction pathway. Activated Arf6 stimulates the formation of lamellipodia at the leading edge of migrating cells by altering at least three signaling pathways that affect actin cytoskeleton organization. Also, partial activated Arf6 may promote neurite outgrowth. Conversely, it is well known that Arf6 depend-endocytosis suppresses 
A new signalsome containing cytohesin-2 and CCDC120 controls neurite outgrowth

neurite outgrowth.

Palacios and D'Souza-Schorey 2003). Also, activated Arf6 controls the localization of cdc42 and subsequent signal regulates cell polarity in glial cells (Osmani et al. 2010, Jayaram et al. 2011). Collectively, activated Arf6 promotes actin formation and remodeling, thereby Arf6 plays an important role in cell morphology, cell migration, and membrane trafficking in non-neuronal cells through various intracellular signal molecules (Figure 1B). Since Arf6 controls Rac1 and/or cdc42 activation also regulates actin dynamic during cell migration as previous studies, we assume that activated Arf6 may regulate activation of downstream effector small GTPases and neurite outgrowth in the same manner, respectively.

1.1 Switching Arfo signal through Arf6GEF and Arf6GAP controls cell motility and membrane trafficking

Accumulating evidences demonstrated that Arf6GEF regulates Arf6 activation and its downstream effectors control activity of various intracellular signal molecules during cell migration (Figure 1B)(Santy and Casanova 2001, Palacios and D'Souza-Schorey 2003, Torii et al. 2010). In particular, it is well known that Arf6GEF cytohesin-1 and cytohesin-2 control cell migration. Also, cytohesin-2 mediated Arf6 activation promotes the recruitment of complex containing Rac-GEF Dock180 and engulfment and cell motility (ELMO) to the leading edge of migrating cells, cytohesin-2/Arf6 signal unit is necessary for Rac mediated cytoskeleton organization as an example (Santy et al. 2005). In addition, the other Arf6-GEF EFA6A (Pleckstrin and Sec7 domain protein) regulates human glioma cells migration and invasion, acting through Arf6-mediated extracellular signal-related kinase (ERK) signaling cascade ( $\mathrm{Li}$ et al. 2006). Based on these findings, Arf6 positive regulator ArfGEF significantly dramatically promotes cell migration via formation of actin cytoskeleton or phosphorylation of MEK/ERK signaling cascade (Tague et al. 2004). Thus, the activation of Arf6GEF contributes to membrane trafficking and cell motility.

On the other hands, Arf6 GTPase-activating protein (GAP), which mainly acts as negative regulator for Arf6, also regulates cell adhesion (Chen et al. 2013), cell migration, membrane traffic, and actin dynamics (de Curtis 2001). Arf-GAP family GIT interacts with paxillin and $\beta$-PIX. The protein also stably forms the complex containing p21-activated kinase (PAK), PAK interacting exchange factor (PIX), and paxillin during cell migration. The PAK-PIX-ArfGAP-paxillin complex is transported from endosome to focal adhesion region at leading edge and participates in formation of focal complex during cell migration (Paris et al. 2003, Brown et al. 2005, Za et al. 2006) and neurite outgrowth (Albertinazzi et al. 2003).

Taken together, both of these enzyme activities are necessary for cell migration through endosomal trafficking systems. Thus, it was confirmed that switching of Arf6 activation through Arf6GEF/Arf6GAP promotes membrane trafficking and recycling of plasma membrane receptor and adhesion molecules (particularly paxillin and integrin) during cell migration.

1.2 Arf6-GEF cytohesin-2 as activator for Arf6 regulate actin dynamics

Cytohesins (also called pleckstrin homology, Sec7 and coiled-coil domains (PSCDs)) are a family of GEF 
A new signalsome containing cytohesin-2 and CCDC120 controls neurite outgrowth

for ARFs that belong to the family of Ras-like small GTPases. All cytohesins are composed of the N-terminal coiledcoil (CC) domain, the catalytic Sec7 domain, the pleckstrin homology domain, and basic amino acids rich region (Figure 1A). The cytohesin family includes cytohesin-1, cytohesin-2/ARNO, cytohesin-3/Grp1, and cytohesin-4 play important roles in regulation of cytoskeletal dynamics by controlling ARFs' activities. Among them, it is well known and demonstrated that cytohesin-2 specific activates Arf6 and/or Arf1 in vitro or in vivo during cell migration (Hongu and Kanaho 2014). Since activated Arf6 promotes small GTPase Rac1 activation in cell migration Arf6 activator cytohesin-2 controls cell migration and actin cytoskeleton organization (Santy and Casanova 2001, Palacios and D'Souza-Schorey 2003, Santy et al. 2005). A positive regulator for Arf6 cytohesin-2 is widely known as key molecule of signal transduction in migrating cells as described above, however, it has not still has been established role for Arf6 activation in neurite outgrowth and characterized its molecular mechanisms particularly upstream molecular mechanisms of cytohesin-2/Arf6 signal unit in in neurons. However, our recent reports described that cytohesin-2 regulates not only cell migration, but also neurite outgrowth in N1E-115 cells (Yamauchi et al. 2009, Torii et al. 2012, Torii et al. 2014).

\subsection{Cytohesin-2 binding proteins}

Much research has identified cytohesin-2 binding proteins and demonstrated some of the specific effects of the interaction between cytohesin-2 and its binding proteins in recent years (Figure 1A). For example, cytohesin-associated scaffold protein (CASP)/Cybr/cytohesin-interacting protein (CYTIP) (Mansour et al. 2002, Tang et al. 2002, Boehm et al. 2003), interaction protein for cytohesin exchange factor (IPCEF)/KIAA0403 (Venkateswarlu 2003), RLIP76/RalBP1 (Lee et al. 2014), ADP-ribosylation factor-like protein 4D (Arl4D) (Hofmann et al. 2007, Li et al. 2007), paxillin (Torii et al. 2010), actinin-1 (Torii et al. 2012) and CCDC120 (Torii et al. 2014) are known as cytohesin interacting protein. The interaction between cytohesin-2 and these proteins also promote cytohesin's GEF activity for Arf6 and actin remodeling, cell migration, and PIP5K through Rac GTPase activation (Figure 1B). In neuronal cells, these activated Arf6 mediated at least two signaling transduction pathways may regulate neurite outgrowth during development. Because cytohesin-2 activity is regulated by complex formation, it may be important to identify and clear the function of Arf6GEF binding partner.

\subsection{Cytohesin-2 mediated Arf6 activation} is necessary for neurite outgrowth

It is well known that cytohesin-2 express in multiple tissues or cells. Our immunofluorescence images using anti-cytohesin-2 antibody, which recognize C-terminal region of cytohesin-2, revealed the expression levels of cytohesin-2 is relatively high in mouse neuroblastoma N1E-115 cells (Torii et al. 2014), DRG neuron, and the others subtype neurons in embryonic day 12.5 (E12.5) mouse. In addition, cytohesin-2 also specifically localizes at growing neurite tips in N1E-115 cells at 24 hours after differentiation induction or growth cone like structures or neurite shafts of N1E-115 cells at 48 hours after differentiation induction. Moreover our previous study demonstrated that cytohesin-2 controls Arf6 activation and neurite outgrowth using specific inhibitor 
of cytohesin family SecinH3 (Fuss et al. 2006, Hafner et al. 2006) or cytohesin-2 siRNA, significantly suppress neurite outgrowth in N1E-115 cells (Torii et al. 2012). Taken together, activated Arf6 and its downstream signal transduction molecules play an important role in neurite outgrowth.

On the other hands, Fluorescence resonance energy transfer (FRET) analysis using Arf6-CyPET and YPET- Golgi-localized $\gamma$-ear containing, Arf-binding protein 3 (GGA3), which specific binds to activated Arf proteins (Boman et al. 2000, DellAngelica et al. 2000), designed by Hall et al. (Hall et al. 2008) reveals that activated Arf6 localized at subcellular regions and basal region of growth cones in neuronal cells (Torii et al. 2012). In addition, FRET live-imaging data exhibits inhibitory effect of cytohesin-2 using SecinH3 dramatically decreased Arf6 activation at plasma membrane. Importantly, the images did not reveal the inhibitory effect at basal region of growth cone (central domain) in neuronal cells. These results indicated that cytohesin-2-Arf6 signal unit mainly is activated at plasma membrane or peripheral domain of growth cone in neurons during development.

Similarly, previous studies described a sec7 domain-containing exchange factor for Arf6 (EFA6A), which is composed by three domains the coiled-coil region, the catalytic Sec7 domain, and the $\mathrm{PH}$ domain, controls membrane recycling and actin cytoskeleton organization (Franco et al. 1999, Derrien et al. 2002, Sakagami et al. 2007). Overexpression of EFA6A promotes neurite outgrowth and controls spine formation mature neuron (Fukaya et al. 2014). Taken together, Arf-GEF dependent Arf6 activation results in cellular morphological changes acting through actin cytoskeleton remodeling and membrane trafficking in neurons as well as in non-neuronal cells.

1.5 Prospective of role for activated Arf6 through cytohesin-2 in neurite outgrowth

So far we have discussed Arf6-cytohesin-2 signaling pathway contributes to multiple aspects of neurite outgrowth mediated by actin cytoskeleton organization in neurons. In addition to that, Ar6 also controls exocytosis from recycle endosome or early endosome to plasma membrane in cells (Balasubramanian et al. 2007). Similarly, recent study demonstrated that Arf6 and its effector PDL2 controls exocytosis of syntenin-1, which interacts with Arf6 and involved in endosomal vesicles and production of exosome (Ghossoub et al. 2014). Since syntenin-1 also interacts with syndecan-4 or Frizzled (Wawrzak et al. 2009), that contribute cell adhesion and cell migration, Arf6 mediated signal pathways including the complexes are necessary for cell migration. Collectively, Arf6-mediated exocytosis manner is important for promoting cell migration, cell adhesion, formation of tight junction. The signaling pathways may be important for neuronal function such as neurite outgrowth in neurons as well as in nonneuronal cells. The mechanism has still not well established yet in neurons, however, technological development of live-imaging or high-resolution microscopy may help us to clear these mechanisms.

Interestingly, Yamauchi et al. also demonstrated that the expression levels of cytohesin-2 and Arl4D are upregulated by valproic acid treatment, that is used as anticonvulsant and mood-stabilizing drug, may coordinately promotes, acting through Arf6, neurite outgrowth via remodeling of the actin cytoskeleton in N1E-115 cells (Yamauchi et al. 2009). The result may be possible to 
A new signalsome containing cytohesin- 2 and CCDC120 controls neurite outgrowth

think that cytohesin-2 is a candidate for target of drug, which impair neuronal diseases or causes neuronal regeneration after nerve injury with disruption of the neuronal axon.

\section{Coiled-coil containing protein 120 (CCDC120)}

CCDC120 does not contain a catalytic domain or a structurally conserved protein domain as far as we can predict. CCDC120 belongs to the CCDC family and composes at least three coiled-coil domains according to protein structural prediction analysis. Other functional regions or catalytic domain in CCDC120 are still not identified.

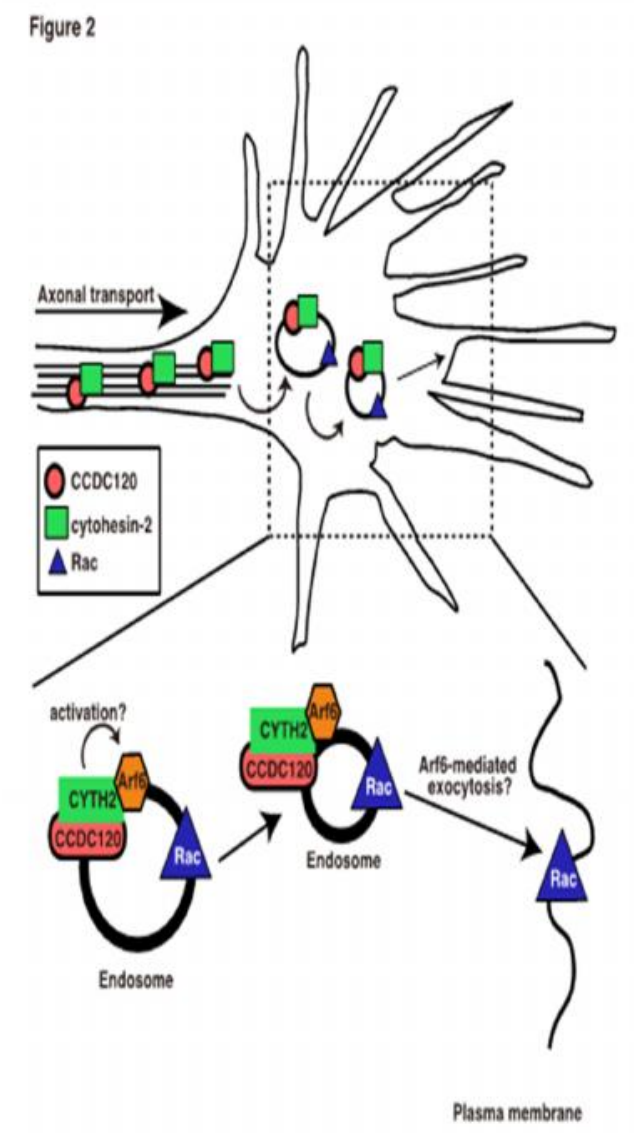

Figure 2.

The hypothesis models for the regulation of cytohesin-2's transport in neurons. Cytohesin-2 is able to colocalize with
CCDC120 in neurite shaft and growth cones of neuron via its $\mathrm{CC}$ domain and the interaction with $\mathrm{CC}$ domain of CCDC120. The complex of cytohesin-2 and CCDC120 may accumulate at early endosome and/or recycling endosome in neurite shaft and growth cone of neurons. When GEF activity is elevated, activated Arf6 promotes translocation of neural vesicle including Rac1, cdc42, or cell adhesion molecule from endosome to plasma membrane. These cytohesin-2 induced localization contribute to neurite outgrowth through small GTPases depend actin cytoskeleton formation and reorganization during development.comprehensive yeast two-hybrid analysis (Rual et al. 2005), however the interaction has not been confirmed yet.

Taken together with prediction analyses and our data suggest that CCDC120 may be a scaffold protein interacts with multiple members of a signaling pathway. Since reduction of CCDC120 by siRNA treatment causes neurite outgrowth defects and promotes formation of short multiple neurite in neuronal cells, we first demonstrated CCDC120 participates in neurite outgrowth (Torii et al. 2014). The result implicates CCDC120 signal pathway contains processes of neurite outgrowth in neurons.

\subsection{CCDC120 as transporter of cytohesin-2}

During neural development, membrane, cytoskeletal, and signaling molecule components are continuously transported along growing neuritis in neurons. Importantly, the large part of vesicles containing CCDC120 reveals immobile, however, approximately $20 \%$ CCDC120 positive vesicles move along neurite from cell soma to cell peripheral 
(anterograde transport) or from cell peripheral to cell soma (retrograde transport) in neuronal cells (Figure 2). Also, CCDC120-cytohesin-2 complex is not colocalized with the vesicle-associated membrane protein 2 (VAMP2)/synaptobrevin 2, VAMP4, VAMP7, Rab8b, or Rab11 at axonal shafts and growth cones. So far, we have not identified the neuronal vesicles containing CCDC120 and cytohesin-2 in neurons yet. In addition, CCDC120 deletion mutant (CCDC120 CC1), which loss cytohesin-2 binding domain (CC1 domain), does not have ability of the recruitment of cytohesin-2 to CCDC120 containing vesicles but does not disrupt neuronal vesicle localization, significantly inhibits neurite outgrowth. Based on these findings, we assume that CCDC120 mediates transport of cytohesin-2 along neurite to facilitate neurite outgrowth. Our report first demonstrated that CCDC120 is responsible for transport of cytohesin-2 along neurite or recruitment of cytohesin-2 to neuronal vesicles (Torii et al. 2014).

Similarly, previous studies demonstrated that a specific GEF of Rab21 Vps9 domain and ankyrin-repeat-containing protein (Varp) is interacts with VAMP7 and sequential axonal transport of vesicles containing the complex mediates neurite outgrowth (Burgo et al. 2009). Taken together, it is certain that small GTPase GEF transports such as retrograde transport or anterograde transport are necessary for neurite outgrowth in neurons.

On the other hands, previous studies showed that cytohesin-2-binding protein Arl4D (Hofmann et al. 2007, Li et al. 2007), which is a member of the ADP-ribosylation factor family of GTP-binding proteins and also phosphorylation of cytohesin-2 at 392 serine residue (S392) by protein kinase $\mathrm{C}$
(PKC) controls cytohesin-2's localization at plasma membrane in cells (Santy et al. 1999). Moreover, it is known that activated Arf6 binds to $\mathrm{PH}$ domain of cytohesin-2 and Grp1 and subsequently recruits cytohesin- 2 to plasma membrane (Cohen et al. 2007). Taken together, $\mathrm{C}$-terminal polybasic region and $\mathrm{PH}$ domain of cytohesin-2 are responsible for its intracellular distribution. To clear the mechanisms of cytohesin-2's translocation in neurons may lead us to an understanding of essential role for Arf6 signal transduction pathways in neurite outgrowth.

\subsection{Post-translational modification of CCDC120}

\subsubsection{Phosphorylation of CCDC120}

CCDC120 localizes at neural vesicles-like structure in growing tips of neurite and mature neurite shafts (Torii et al. 2014). Jungmichel et al also demonstrated that CCDC120 is transported from intracellular region to plasma membrane by Platelet-Derived Growth Factor (PDGF) stimulation through Akt (also known as Protein kinase $\mathrm{B} \quad(\mathrm{PKB})$ )-mediated serine/threonine phosphorylation in non-neuronal cells (Figure 3A) (Jungmichel et al. 2014). It is still largely unknown whether the phosphorylation of CCDC120 is necessary for its axonal transport along neurites or switches signal transduction pathways in neurons. The serine/threonine phosphorylation of CCDC120 may also play a role in interaction with cyothesin-2 or other signal molecules and control neurite outgrowth. Thus, it will be interesting to identify the phosphorylation site of CCDC120 to demonstrate the effects of neuronal function. 
A new signalsome containing cytohesin-2 and CCDC120 controls neurite outgrowth

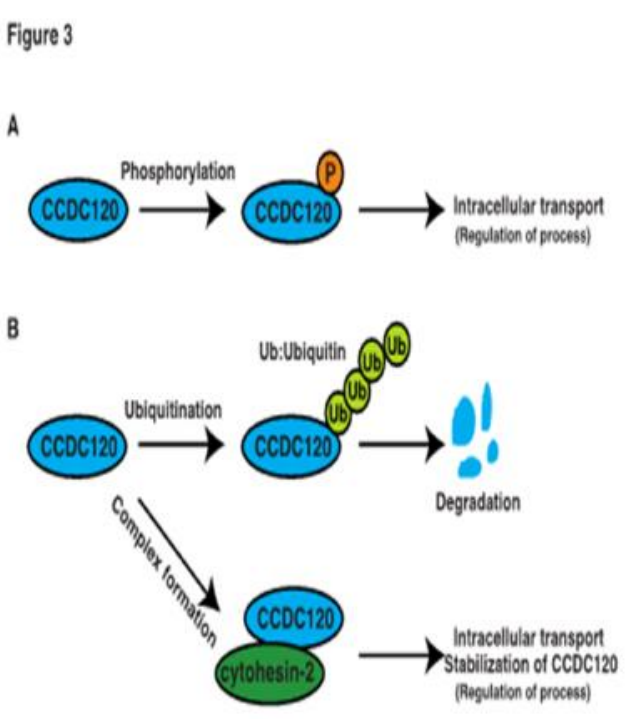

Figure 3.

Post-translational processing of CCDC120. Phosphorylation and ubiquitination of CCDC120 may serve cellular processes in cells. (A) CCDC120 is phosphorylated by $\mathrm{Akt}$ and the phosphorylation regulates its intracellular transport. (B) $\mathrm{CCDC} 120$ is also ubiquitylated and the modified CCDC120 is degradated subsequently. The proteolysis of CCDC120 is suppressed by complex formation between CCDC120 and cytohesin- 2 .

\subsubsection{Ubiquitination of CCDC120}

Most of biological processes rely on functional protein modules in various cases. Post-translational modifications (PTMs) of proteins such as phosphorylation, acetylation, glycosylation, methylation, ubiquitination (Clague and Urbé 2010), and sumoylation represent an important level of cellular control and regulate their cellular distribution. It is also known that ubiquitination signal transduction molecules regulates neurite outgrowth (Hamilton and Zito 2013). Importantly, the poly-ubiqutination of CCDC120 and subsequent its degradation was observed.
It is therefore that the ubiquitin-proteosome system mediates CCDC120 degradation. However, the proteolysis of CCDC120 through ubiquitin-proteosome pathway is inhibited by the complex formation between cytohesin-2 and CCDC120 as shown in Figure 3B (Torii et al. 2014). The findings suggest that the complex formation between CCDC120 and cytohesin-2 may regulate a stabilization of CCDC120 or block proteolysis in ubiqutination-dependent manner. The ubiquitination of CCDC120 also may paly an important role in its axonal transport in neurons. The clarification of role for CCDC120's post-translational modification in neurons provides understanding of its neuronal function.

2.3 Prospective of role for CCDC120 in neurite outgrowth

We characterized CCDC120 also accumulates neuronal vesicles. In addition, CCDC120 were as cytohesin-2 activator and/or transporter. CCDC120 deletion mutant significantly disrupts neuronal vesicle localization and inhibits neurite outgrowth in neuronal cells (Torii et al. 2014). Moreover, CCDC120 positive vesicles also include activated Arf6. We hypothesize that the activated Arf6 regulates transport of vesicles containing small GTPases and/or cell adhesion molecules from recycling endosomes to the cortical region of the cells (Figure 2). Neuronal vesicles containing CCDC120 is still not identified and characterized completely yet. In order to gain deeply understanding, studies to identify CCDC120 binding protein using affinity chromatography and co-immunostaining using various neuronal vesicle markers may be needed.

\section{CONCLUSIONS}

Based on our studies (Torii et al. 
2012, Torii et al. 2014), cytohesin-2 was demonstrated as positive regulator of neurite outgrowth and an unknown function CCDC120 was first characterized as cytohesin-2's transporter and/or activator (Torii et al. 2014). In this review, we will emphasize that cytohesin-2-Arf6-CCDC120 complex signal unit is necessary for neurite outgrowth and CCDC120 also controls recruitment cytohesn-2 to neuronal vesicles during neuronal development. Also, activated Arf6 by cytohesin-2-CCDC120 complex formation may play an important role in Rac activation or recruitment of Rac to plasma membrane, regulates neurite outgrowth. The Further understanding of the multistep processes through signal pathways including CCDC120 in neurite outgrowth will provide additional insight into how upstream signal protein of cytoohesin-2 may affect Arf6 activation and subsequent neurite outgrowth through Rac. Although the paramount importance of Arf6/cytohesin-2 signaling pathways in neurite outgrowth, many fundamental questions remain unresolved.

Although some studies have been in vitro, data collected from in vivo studies still needs to understand of a new signalsome containing cytohesin-2 and CCDC120 in physiological systems. Therefore, more research using knockout mouse and transgenic mouse of these proteins is needed to determine exact neuronal function of cytohesin-2. As described above, cytohesin- 2 expresses in various cells and tissues, however, the expression levels of cytohesin-2 in neuron relatively higher than other cells in central nervous systems and peripheral nervous systems. Using neuron specific promoter regulated cytohesin-2 conditional knockout mice (cKO) or CCDC120 cKO mice, roles of cytohesin-2-Arf6 signal in neurons may be cleared in the near future.
These mice may exhibit disruption of axon pathfinding (axon guidance), branching, or neuron-neuron network. Moreover, the disruption of neurite outgrowth by loss of cytohesin- 2 may be able to be partially rescued by overexpression of cytohesin-2 using in utero electroporation or crossing with transgenic mice expressing cytohesin-2 wild type.

Finally, we and others recently demonstrated physiological roles for Arf6 in myelination using knockout mice of Arf6 (Akiyama et al. 2014) and Arf6GEF (Yamauchi et al. 2012, Torii et al. 2015). Conditional knockout mice of cytohesin-2, which is controlled by the major myelin marker myelin protein zero (MPZ), were generated recently. Previous our study demonstrated that the cytohesin-2 knockout mice exhibit hypomyelination in peripheral nervous systems (Torii et al. 2015). Moreover, a transgenic mouse expressing microRNA adapted short hairpin RNA (shRNA-mir) of Arf6 under the control of the MPZ promoter also reveals hypomyelination in peripheral nervous systems (Yamauchi et al. 2012). These studies may help contribute to the discovery of future therapies for patients with Charcot-Marie-Tooth (CMT) disease, is inherited peripheral neuropathy or decrease the thickness of myelin. Thus, it is clearly that the other cytohesin family protein cytohesin-1, is a GEF for Arf6, also controls myelin thickness (Yamauchi et al. 2012). Continued investing into unresolved issues of cytohesin family has the potential to identify targets for the therapeutic manipulation of neurite outgrowth or myelination in disease states.

\section{ACKNOWLEDGEMENTS}

This work was supported by Grants-in-Aid both for Basic Scientific Research from the Japanese Ministry of 
A new signalsome containing cytohesin-2 and CCDC120 controls neurite outgrowth

Education, Culture, Sports, Science, and Technology (MEXT) and for Medical Scientific Research from the Japanese Ministry of Health, Labor, and Welfare (MHLW). This work was also supported by the Innovative Areas' Scientific Research (Glial Assembly) and the Takeda Science Foundation. This work was supported by Grants-in-Aid both for
Basic Scientific Research from the Japanese Ministry of Education, Culture, Sports, Science, and Technology (MEXT) and for Medical Scientific Research from the Japanese Ministry of Health, Labor, and Welfare (MHLW). This work was also supported by the Innovative Areas' Scientific Research (Glial Assembly) and the Takeda Foundation. 


\section{REFERENCES}

Akiyama M., H. Hasegawa, T. Hongu, M. A. Frohman, A. Harada, H. Sakagami, and Y. Kanaho. 2014. "Trans-regulation of ologodendrocyte myelination by neurons through small GTPase Arf6-regulated secretion of fibroblast growth factor-2." Nat Commun 5:4744. doi: $10.1038 /$ ncomms 5744 .

Albertinazzi, C., L. Za, S. Paris, and I. de Curtis. 2003. "ADP-ribosylation factor 6 and a functional PIX/p95-APP1 complex are required for Rac1B-mediated neurite outgrowth." Mol Biol Cell 14(4):1295-307.

Balasubramanian, N., D. W. Scott, J. D. Castle, J. E. Casanova, and M. A. Schwartz. 2007. "Arf6 amd microtubules in adhesion-dependent trafficking of lipid rafts." Nat Cell Biol 9(12):1381-91.

Boehm, T., S. Hofer, P. Winklehner, B. Kellersch, C. Geiger, A. Trockenbacher, S. Neyer, H. Fiegl, S. Ebner, L. Ivarsson, R. Schneider, E. Kremmer, C. Heufler, and W. kolanus. 2003. "Attenuation of cell adhesion in lymphocytes is regulated by CYTIP, a protein which mediates signal complex sequestration." EMBO J 22(5):1014-24.

Boman, A. L., C. J. Zhang, X. Zhu, and R. A. Kahn. 2000. "A family of ADP-ribosylation factor effectors that can alter membrane transport through the trans-Golgi." Mol Biol Cell 11(4):1241-55.

Brown, M. C., L. A. Cary, J. S. Jamieson, J. A. Cooper, and C. E. Turner. 2005. "Src and FAK kinases cooperate to phosphorylate paxillin kinase linker, stimulate its focal adhesion localization, and regulate cell spreading and protrusiveness." Mol Biol Cell
16(9):4316-28.

Burd, C. G., T. I. Strochlic, and S. R. Gangi Setty. 2004. "Arf-like GTPases: not so Arf-like after all." Trends Cell Biol 14(12):687-94.

Burgo, A., E. Sotirakis, M. C. Simmier, A. Verraes, C. Chamot, J. C. Simpson, L. Lanzetti, V. Proux-Gillardeaux, and T. Galli. 2009. "Role of Varp, a Rab21 exchange factor and TI-VAMP/VAMP7 partner, in neurite outgrowth." $E M B O$ Rep 10(10):1117-24. doi: 10.1038/embor.2009.186.

Chardin, P., S. Paris, B. Antonny, S. Robineau, S. Béraud-Dufour, C. L. Jackson, M. Chabre. 1996. "A human exchange factor for ARF contains Sec7and placekstrin-homology domains." Nature 384(6608):481-4.

Chen, P. W., X. Jian, H, Y. yoon, and P. A. Randazzo. 2013. "ARAP2 signals through Arf6 and Rac1 to control focal adhesion morphology." $J$ Biol Chem 288(8):5849-60. doi: 10.1074/jbc.M112.415778.

Chesneau, L., D. Dambournet, M. Machicoane, I. Kouranti, M. Fukuda, B. Goud, and A. Echard. 2012. "An ARF6/Rab35 GTPase cascade for endocytic recycling and successful cytokinesis." Curr Biol 22(2):147-53. doi: 10.1016/j.cub.2011.11.058.

Clague, M. J., and S. Urbé. 2010. "Ubiquitin: same molecule, different degradation pathways." Cell 143(5):682-685. doi: 10.1016/j.cell.2010.11.012.

Cohen, L. A., A. Honda, P. Varnai, F. D. Brown, T. Balla, and J. G. Donaldson. 2007. "Active Arf6 recruits 
ARNO/cytohesin GEFs to the PM by binding their PH domains. Mol Biol Cell 18(6):2244-53.

Craig, A. M., and G. Banker. 1994. "Neuronal polarity." Annu Rev Neurosci 17:267-310.

da Silva, J. C, and C. G. Dotti. 2002. "Breaking the neuronal sphere: regulation of the actin cytoskeleton in neuritogenesis. Nat Rev Neurosci 3(9):694-704.

de Curtis, I. 2001. "Cell migration: Gaps between membrane traffic and the cytoskeleton." EMBO Rep 2(4):277-81.

Dell'Angelica, E. C., R. Puertollano, C. Mullins, R. C. Aguilar, J. D. Vargas, L. M. Hartnell, and J. S. Bonifacino. 2000. "GGAs: a family of ADP ribosylation factor-binding proteins related to adaptors and associated with the Golgi complex." J Cell Biol 149(1):81-94.

Derrien, V., C. Couillaut, M. Franco, S. Martinea, P. Montcourrier, R. Houlgatte, and P. Chavrier. 2002. "A conserved C-terminal domain of EFA6-family ARF6-guanine nucleotide exchange factors induces lengthening of microvilli-like membrane protrusions. $J$ Cell Sci 115(Pt 14):2867-79.

Donaldson, J. G. 2003. "Multiple roles for Arf6: sorting, structuring, and signaling at the plasma membrane. $J$ Biol Chem 278(43):41573-6.

D'Souza-Schorey C, and P. Chavrier. 2006. "ARF proteins: roles in membrane traffic and beyond. Nat Rev Mol Cell Biol. 7(5):347-58.

Franco, M., P. J., Peters, J. Boretto, E. van Donselaar, A. Neri, C. D'Souza-Schorey, and P. Chavrier. 1999. "EFA6, a sec7 domain-containing exchange factor for ARF6, coordinates membrane recycling and actin cytoskeleton organization." EMBO J 18(6):1480-91.

Frank, S., S. Upender, S. H. Hansen, J. E. Casanova. 1998. "ARNO is a guanine nucleotide exchange factor for ADP-ribosylation factor 6." $\mathrm{J} \mathrm{Biol} \mathrm{Chem}$ 273(1):23-27.

Frank, S. R., J. C. Hatfield, and J. E. Casanova. 1998. "Remodeling of the actin cytoskeleton is coordinately regulated by protein kinase $\mathrm{C}$ and the ADP-ribosylation factor nucleotide exchange factor ARNO." Mol Biol Cell 9(11):3133-46.

Fukaya, M., D. Fukushima, Y. Hara, and H. Sakagami. 2014. "EFA6A, a guanine nucleotide exchange factor Arf6, interacts with sorting nexin-1 and regulates neurite outgrowth." J Neurochem 129(1):21-36. doi: 10.1111/jnc.12524.

Fuss, B., T. Becker, I Zinke, and M. Hoch. 2006. "The cytohesin Steppke is essentiall for insulin signaling in Drosophila." Nature 444(7121):945-8.

Gallo, G. 2011. "The cytoskeletal and signaling mechanisms of axon collateral branching." Dev Neurobiol 71(3):201-20. doi: 10.1002/dneu.20852.

Ghossoub, R., F. Lembo, A. Rubio, C. B. Gaillard, J. Bouchet, N. Vitale, J. Slavík, M. Machala, and P. Zimmermann. 2014. "Syntenin-ALIX exosome biogenesiss and budding into multivesicular bodies are controlled by ARF6 and PLD2. Nat Commun 5:3477. doi: 10.1038/ncomms4477. 
A new signalsome containing cytohesin-2 and CCDC120 controls neurite outgrowth

Govek, E. E., S. E. Newey, and L. Van Aelst. 2005. "The role of the Rho GTPases in neuronal development." Gene Dev 19(1):1-49.

Hafner, M., A. Schmitz, I. Grüne, S. G. Srivatsan, B. Paul, W. Kolanus, T. Quast, E. Kremmer, I. Bauer, and M. Famulok. 2006. "Inhibition of cytohesins by SecinH3 leads to heaptic insulin resistance. Nature 444(7121):941-4.

Hall, B., M. A. McLean, K. Davis, J. E. Casanova, S. G. Sligar, and M. A. Schwartz. 2008. "A fluorescence resonance energy transfer activation sensor for Arf6." Anal Biochem 374(2):243-9.

Hamilton, A. M. and K. Zito. 2013. "Breaking it down: the ubiquitin proteasome system in neuronal morphogenesiss." Neural Plast 2013:196848. doi: 10.1155/2013/196848.

Hancock, W. O. 2014. "Bidirectional cargo transport: moving beyond tug of war." Nat Rev Mol Cell Biol 15(9):615-28. doi: 10.1038/nrm3853.

Hirokawa N. 1997. "The mechanisms of fast and slow transport in neurons: identification and characterization of the new kinesin superfamily motors." Curr Opin Neurobiol 7(5):605-14.

Hofmann I, A. Thompson, C. M. Sanderson, and S. Munro. 2007. "The Arl4 family of small $\mathrm{G}$ proteins can recruit the cytohesin Arf6 exchange factors to the plasma membrane." Curr Biol 17(8):711-6.

Hongu, T., and Y. Kanaho. 2014, "Activation machinery of the small GTPase Arf6." Adv Biol Regul 54:59-66. doi: 10.1016/j.jbior.2013.09.014.
Jaworski, J. 2007. "ARF6 in the nervous system." Eur J Cell Biol 86(9):513-24.

Jayaram, B., I. Syed, C. N. Kyathanahalli, C. J. Rhodes, and A. Kowluru. 2011. "Arf nucleotide binding site opener [ARNO] promotes sequential activation of Arf6, Cdc42, and Rac1 and insulin secretion in INS 832/13 $\beta$-cells and rat islets." Biochem Pharmacol 81(8):1016-27. doi: 10.1016/j.bcp.2011.01.006.

Jungmichel, S., K. B. Sylvestersen, C. Choudhary, S. Nguyen, M. Mann, and M. L. Nielsen. 2014. "Specificity and commonality of the phosphorinositide-binding proteome analyzed by quantitateive mass spectrometry." Cell Rep 6(3):578-91. doi: 10.1016/j.celrep.2013.12.038.

Kahn, R. A., J. Cherfils, M. Elias, R. C. Lovering, S. Munro, and A. Schurmann. 2006. "Nomenclature for the human Arf family of GTP-binding proteins: ARF, ARL, and SAR proteins." J Cell Biol 172(5):645-50.

Kobayashi, H. K. Etoh, and M. Fukuda. 2014a. "Rab35 is translocated from Arf6-positive perinuclear recycling endosomes to neurite tips during neurite outgrowth." Small GTPases 5:e29290. doi: 10.4161/sgtp.29290.

Kobayashi, H., K. Etoh, N. Ohbayashi, and M. Fukuda. 2014b. "Rab35 promotes the recruitment of Rab8, Rab13 and Rab36 to recycling endosomes through MICAl-L1 during neurite outgrowth." Biol Open 3(9):803-14. doi: 10.1242/bio.20148771.

Lee, S., J. G. Wurtzel, and L. E. Goldfinger. 2014. "The RLIP76 N-terminal binds ARNO to regulate PI 
A new signalsome containing cytohesin-2 and CCDC120 controls neurite outgrowth

3-kinase, Arf6 and Rac signaling, cell spreading and migration." Biochem Biophy Res Commun 454(4):560-5. doi: 10.1016/j.bbrc.2014.10.114.

Li, C. C., T. C. Chiang, T. S. Wu, G. Pachero-Rodriguez, J. Moss, and F. J. Lee. $2007 . \quad$ "ARL4D recruits cytohesin-2/ARNO to modulate actin remodeling." $\mathrm{Mol}$ Biol Cell 18(11):4420-37.

Li, M., S. S. Ng, J. Wang, L. Lai, S. Y. Leung, M. Franco, Y. Peng, M. L. He, H. F. Kung, and M. C. Lin. 2006. "EFA6A enhances glioma cell invasion through ADP ribosylation factor 6/extracellular signal-regulated kinase signaling." Cancer Res 66(3):1583-90.

Mansour, M. S. Y. Lee, and B. Pohajdak. 2002. "The N-terminal coiled coil domain of the cytohesin/ARNO family of guanine nucleotide exchange factors interacts with the scaffolding protein CASP." J Biol Chem 277(35):32302-9.

Mattila, P. K., and Lappalainen, P. 2008. "Filopodia: moleculer architecture and cellular functions." Nat Rev Mol Cell Biol 9(6):446-54. doi: 10.1038/nrm2406.

Miki H., M. Setou, K. kaneshiro, and N. Hirokawa. 2001. "All kinesin superfamily protein, KIF, genes in mouse and human. Proc Natl Acad Sci $U$ S A 98(13):7004-11.

Miyamoto, Y., N. Yamamori, T. Torii, A. Tanoue, and J. Yamauchi. 2014. "Rab35, acting through ACAP2 switching off Arf6, negatively regulates oligodendrocyte differentiation and myelination." Mol Biol Cell 25(9):1532-42. doi: 10.1091/mbc.E13-10-0600.
Osmani, N. F. Peglion, P. Chavrier, and S. Etienne-Manneville. 2010. "Cdc42 localization and cell polarity depend on membrane traffic." $J$ Cell Biol 191(7):1261-9. doi: 10.1083/jcb.201003091.

Palacios, F., and C. D'Souza-Schorey. 2003. "Modulation of Rac1 and ARF6 activation during epithetial cell scattering." $J$ Biol Chem 278(19):17395-400.

Paris, S., R. Longhi, P. Santambrogio, and I. de Curtis. 2003. "Leucine-zipper-mediated homo- and hetero-dimerization of GIT family p95-ARF GTPase-activating protein, PIX-, paxillin-interacting proteins 1 and 2." Biochem J 372(Pt 2):391-8.

Pelletán L. E., L. Suhaiman, C. C. Vaquer, M. A. Bustos, G. A. De Blas, N. Vitale, L. S. Mayorga, and S. A. Belmonte. "ADP ribosylation factor 6 (ARF6) promotes acrosomeal exocytosis by modulating lipid turnover and Rab3A activation." $J$ Biol Chem 290(15):9823-41. doi: 10.1074/jbc.M114.629006.

Raiborg, C., E. M. Wenzel, N. M. Pedersen, H. Olsvik, K. O. Schink, S. W. Schultz, M. Vietri, V. Nisi, C. Bucci, A. Brech, T. Johansen, and H. Stenmark. 2015. "Repeated ER-endosome contacts promote endosome translocateion and neurite outgrowth." Nature 520(7546):234-8. doi: 10.1038 /nature14359.

Rual, J. F., K. Venkatesan, T. Hao, T. Hirozane-Kishikawa, A. Dricot, N. Li, G. F. Berriz, F. D. Gibbons, M. Dreze, N. Ayivi-Guedehoussou, N. Klitgord, C. Simon, M. Boxem, S. Milstein, J. Rosenberg, D. S. Goldberg, L. V. Zhang, S. L. Wong, G. Franklin, S. Li, J. S. Albala, J. 
A new signalsome containing cytohesin-2 and CCDC120 controls neurite outgrowth

Lim, C. Fraughton, E. Llamosas, S. Cevik, C. Bex, P. Lamesch, R. S. Sikorski, J. Vandenhaute, H. Y. Zoghbi, A. Smolyar, S. Bosak, R. Sequerra, L. Doucette-Stamm, M. E. Cusick, D. E. Hill, F. P. Roth, and M. Vidal. 2005. "Towards a proteome-scale map of thehuman protein-protein interaction network." Nature 437(7062):1173-8.

Sabe, H. 2003. "Requirement for Arf6 in cell adhesion, migration, and cancer cell invasion." J Biochem 134(4):485-9.

Sakagami, H., T. Honma, J. Sukegawa, Y. Owada, T. Yanagisawa, and H. Kondo. 2007. "Somatodendritic localization of EFA6A, a guanine nucleotide exchange factor 6 , and its possible interaction with alpha-actinin in dendritic spines." Eur $J$ Neurosci 5(3):618-28.

Santy, L. C., S. R. Frank, J. C. Hatfield, and J. E. Casanova. 1999. "Regulation of ARNO nucleotide exchange by a $\mathrm{PH}$ domain electrostatic switch." Curr Biol 9(20):1173-6.

Santy, L. C., and J. E. Casanova. 2001. "Activation of ARF6 by ARNO stimulates epithelial cell migrateon through downstream activation of both Rac1 and phospholipase D." J Cell Biol 154(3):599-610.

Santy, L. C., K. S. Ravichandran, and J. E. Casanova. 2005. "The Dock180/Elmo complex couples ARNO-mediated Arf6 activation to the downstream activation of Rac1." Curr Biol 15(19):1749-54.

Stenmark, H. 2009. "Rab GTPases as coordinators of vesicle traffic." Nat Rev Mol Cell Biol 10(8):513-25. doi: 10.1038/nrm2728.
Tague S. E., V. Muralidharan, and C. D'Souza-Schorey. 2004. "ADP-ribosylatio factor 6 regulates tumor cell invasion through the activation of the MEK/ERK signaling pathway." Proc Natl Acad Sci U S A 101(26):9671-6.

Tang, P., T. P. Cheng, D. Agnello, C. Y. Wu, B. D. Hissong, W. T. Watford, H. J. Ahn, J. Galon, J. Moss, M. Vaughan, J. J. O'Shea, and M. Gadina. 2002. "Cybr, a cytokine-inducible protein that bids cytohesin-1 and regulates its activety." Proc Natl Acad sci U S A 99(5):2625-9.

Torii, T., Y. Miyamoto, K. Tago, K. Sango, K. Nakamura, A. Sanbe, A. Tanoue, and J. Yamauchi. 2014. "Arf6 guanine nucleotide exchange factor cytohesin- 2 binds to $\mathrm{CCDC} 120$ and is transported along neurites to mediate neurite outgrowth." J Biol Chem 289(49): 33887-903. doi: 10.1074/jbc.M114.575787.

Torii, T., Y. Miyamoto, K. Nakamura, M. Maeda, J. Yamauchi, A. Tanoue. 2012. "Arf6 guanine nucleotide exchange factor, cytohesin-2, interacts with actinin-1 to regulate neurite outgrowth." Cell Signal 24(9):1872-82. doi: 10.1016/j.cellsig.2012.05.016.

Torii, T., Y. Miyamoto, A. Sanbe, K. Nishimura, J. Yamauchi, and A. Tanoue. 2010. "Cytohesin-2/ARNO, through its interaction with focal adhesion adaptor protein paxillin, regulates preadipocyte migration via the downstream activation of Arf6." J Biol Chem 285(31):24270-81. doi: 10.1074/jbc.M110.125658.

Torii, T., N. Ohno, Y. Miyamoto, K. Kawahara, Y. Saitoh, K. Nakamura, S. Takashima, H. Sakagami, A. Tanoue, and J. Yamauchi. 2015. "Arf6 guanine-nucleotide exchange factor 
A new signalsome containing cytohesin-2 and CCDC120 controls neurite outgrowth

cytohesin-2 regulates myelination in nerves." Biochem Biophy Res Commun 460(3):819-25.

doi:

10.1016/j.bbrc.2015.03.113.

van den Bosch M. T., A. W. Poole, and I. Hers. 2014. "Cytohesin-2 phosphorylation by protein kinase $\mathrm{C}$ relieves the constitutive suppression of platelet dense granule secretion by ADP-ribosylation factor 6." $J$ Thromb Haemost 12(5):726-35. doi: $10.1111 /$ jth. 12542 .

Venkateswarlu K. 2003. "Interaction protein for cytohesin exchange factor 1 (IPCEF1) binds cytohesin 2 and modifies its activity." J Biol chem 279(8):6205-8.

Villarroel-Campos, D., L. Gastaldi, C. conde, A. Caceres, and C. Gonzalez-Billault. 2014. "Rab-mediated trafficking role in neurite formation" $J$ Neurochem 129(2):240-8. doi: 10.1111/jnc. 12676.

Wawrzak, D., A. Luyten, K. Lambaerts, and P. Zimmermann. 2009. "Frizzled-PDZ scaffold interactions in the control of Wnt signaling." $\quad A d v \quad$ Enzyme Regul 49(1):98-106. doi: 10.1016/j.advenzreg.2009.01.002.

Yamamoto H., H. Koga, Y. Katoh, S. Takahashi, K. Nakayama, and H. W. Shin. 2010. "Functional cross-talk between Rab14 and Rab4 throgh a dual effector, RUFY1/Rabip4." Mol Biol Cell 21(15):2746-55. doi: 10.1091/mbc.E10-01-0074.
Yamauchi, J., Y. Miyamoto, T. Torii, R. Mizutani, K. Nakamura, A. Sanbe, H. Koide, S. Kusakawa, and A. Tanoue. 2009. "Valproic acid-inducible Arl4D and cytohesin-2/ARNO, acting through the downstream Arf6, regulate neurite outgrowth in N1E-115 cells." Exp Cell Res 315(12):2043-52. doi: 10.1016/j.yexcr.2009.03.012.

Yamauchi, J., Y. Miyamoto, T. Torii, S. Takashima, K. Kondo, K. Kawahara, N. Nemoto, J. R. Chan, G. Tsujimoto, and A. Tanoue. 2012. "Phosphorylation of cytohesin-1 by Fyn is required for initiateon of myelination and the extent of myelination during development." Sci Signal 5(243):ra69.

Yap, C. C. and B. Winckler. 2012. " Harnessing the power of the endosome to regulate neural development." Neuron 74(3):440-51. doi: 10.1016/j.neuron.2012.04.015.

Za, L., C. Albertinazzi, S. Paris, M. Gagliani, C. Tacchetti, and I. de Curtis. 2006. "betaPIX controls cell motility and neurite extension by regulating the distribution of GIT1." J Cell Sci 119(Pt 13):2654-66. 